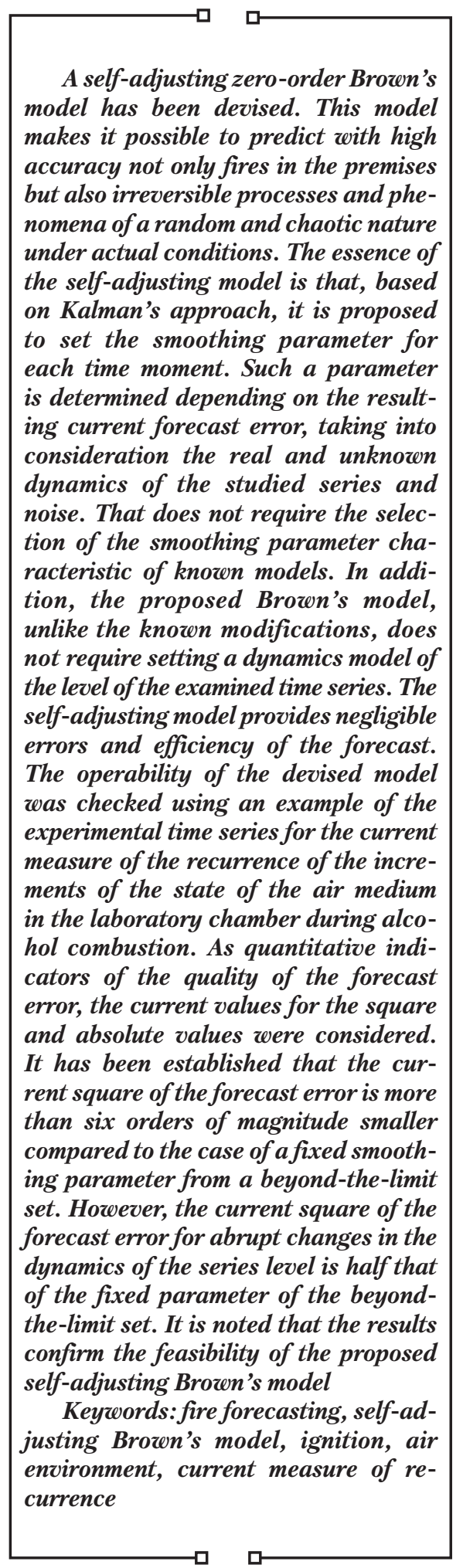

\title{
DEVISING A SELF-ADJUSTING ZERO-ORDER BROWN'S MODEL FOR PREDICTING IRREVERSIBLE PROCESSES AND PHENOMENA
}

Boris Pospelov

Doctor of Technical Sciences, Professor Scientific-Methodical Center of Educational Institutions in the Sphere of Civil Defence O. Honchara str., 55, Kyiv, Ukraine, 01601

VIadimir Andronov

Doctor of Technical Sciences, Professor*

Evgeniy Rybka

Corresponding author

Doctor of Technical Sciences, Senior Researcher*

E-mail: e.a.ribka@gmail.com

Olekcii Krainiukov

Doctor of Geographical Sciences, Associate Professor Department of Environmental Safety and Environmental Education**

Nadi ya Maks ymenko

Doctor of Geographical Sciences, Professor Department of Environmental Monitoring and Management**

I gor B i r y u k o v

Doctor of Technical Sciences, Associate Professor Department of Missile and Artillery Weapons***

Maxim Zhuravskij

$\mathrm{PhD}$, Associate Professor Department of Organization of Educational Activities of the Educational and Methodical Center $* * * *$

Yuli i a B zuhla

$\mathrm{PhD}$, Associate Professor Department of Prevention Activities and Monitoring****

Ihor Morozov

$\mathrm{PhD}$, Senior Researcher Department of Research and Organization***

I hor Yevtushenko $\mathrm{PhD}$

Department of Tactics-special, Fire and Special Physical Trainingjuridical Personnel Training Yaroslav Mudryi National Law University Pushkinska str., 77, Kharkiv, Ukraine, 61024 * Research Center

National University of Civil Defence of Ukraine Chernyshevska str., 94, Kharkiv, Ukraine, 61023

$* * V$. N. Karazin Kharkiv National University Svobody sq., 4, Kharkiv, Ukraine, 61022 ***National Academy of the National Guard of Ukraine Zakhysnykiv Ukrainy sq., 3, Kharkiv, Ukraine, 61001 $\star * * *$ National University of Civil Defence of Ukraine

Chernyshevska str., 94, Kharkiv, Ukraine, 61023

Received date 26.08.2021 Accepted date 11.10.2021 Published date 29.10.2021
How to Cite: Pospelov, B., Andronov, V., Rybka, E., Krainiukov, O., Maksymenko, N., Biryukov, I., Zhuravskij, M., Bezuhla, Y., Morozov, I., Yevtushenko, I. (2021). Devising a self-adjusting zero-order brown's model for predicting irreversible processes and phenomena. Eastern-European Journal of Enterprise Technologies, 5 (10 (113)), $40-47$. doi: https://doi.org/10.15587/1729-4061.2021.241474

\section{Introduction}

Forecasting $(\mathrm{F})$ is considered one of the significant stages in resolving the issue of ensuring sustainable and effective development of the socio-economic life of society. The basis of $\mathrm{F}$ is usually the models of predicted processes and phenomena (PPs). All PPs can be categorized into two main classes - reversible and irreversible. Models of reversible PPs 
are based on the stability of their certain probabilistic characteristics over time. Therefore, the basis of reversible PPs $\mathrm{F}$ is usually the analysis of past states and their subsequent extrapolation. In this regard, the models of reversible PPs are described on the basis of representations accepted in probability theory and mathematical statistics. For irreversible PPs, the stability of probabilistic characteristics evolves over time or may be absent at all. This significantly complicates the $\mathrm{F}$ of irreversible PPs and creates the task of developing self-adjusting models for them. The relevance of this issue is further determined by the fact that most of the real PPs that violate the sustainable development of the sphere of socio-economic life of society are irreversible. An important type of an actual irreversible PP is fire. Fire can occur both in various ecosystems [1] and at production facilities [2,3]. The most dangerous ones are the fires in the premises (FP) [4]. This is explained by the fact that FPs are massive and cause significant damage not only to human life [5] but also to objects [6], as well as the environment [7]. More than 90,000 lives are claimed by FPs each year, and the material damage they cause is measured in trillions of dollars. In this regard, the task of devising effective models for the $\mathrm{F}$ of irreversible PPs becomes particularly relevant.

\section{Literature review and problem statement}

It is known that the tendencies of irreversible PPs change over time (are evolutionary in nature) or are absent at all (have a chaotic character). The F of such PPs are extremely difficult. The best result for this type of PPs is given by adaptive models. A widely used in various applications is the adaptive zero-order Brown's model (ZOBM) [8]. Following the ZOBM, F is determined through the previous forecast, which is corrected by the deviation of the current observation from the previous forecast. This ensures that the $\mathrm{ZOBM}$ is adapted to new incoming information. ZOBM is a one-parameter adaptive model whose properties are determined by the value of $h$, the smoothing parameter, which acts as a weight for the current corrective deviation. In general, ZOBM is used for the $\mathrm{F}$ of stationary [9] and evolutionary PPs [10]. For stationary PPs, $h$ takes fixed values from the classical set between 0 and 1 . In this case, the specific value of the fixed parameter $h$ is proposed to be selected experimentally for each type of a time series (TS). There are more than a dozen ways to select a parameter from the classical set [11]. In [8], it is proposed to determine the value of $h$ experimentally, based on minimizing the sum of squares of current errors in $\mathrm{F}$. The limitations of this technique include the need to repeatedly determine the forecast for the same TS data at different fixed values of $h$ and calculate the sum of the squares of the current $\mathrm{F}$ errors. That means that the technique from [8] is complex and does not allow it to be implemented in real time for the F of PPs of an evolutionary nature. The development of a self-adjusting ZOBM and the smoothing parameter are not considered or investigated in [8-11]. In $[12,13]$, it is proposed to determine the value of $h$ in accordance with the inequality $0.1<h<0.3$. This range is not justified by anything. At the same time, those recommendations are limited mainly to the $\mathrm{F}$ of stationary TS with the presence of a clear trend. It is noted that in most cases good F results occur at $h=1$. However, if $h<0.3$ are preferred, this is a sign of the non-stationarity of TS, and indicates the need for more complex models. In [14], it is noted that with a suf- ficiently large dispersion of noise, the values of $h$ should not exceed 0.2. At the same time, for each specific TS, the choice of the value of $h$ should be made taking into consideration the goal of F. In [8], it is noted that the solution to the problem of choosing the optimal value of $h$ is associated with an assessment of the current accuracy of F. If the high accuracy of $\mathrm{F}$ is provided at values of $h$ close to 1 , then this indicates the incorrectness of the corresponding ZOBM. At the same time, a self-adjusting ZOBM is not considered. For the F of PPs of evolutionary nature, paper [15] recommended choosing the values for the parameter $h$ in accordance with the inequality $0<h<1$, and for PPs of a chaotic nature $-1<h<2$. It is indicated that for each specific TS there is an optimal value of the parameter $h$, determined by the properties of the predicted TS. There is also an approach reported in [16], based on an analytical solution to the inverse problem $h=2 /(k+1)$, where $k$ is the number of steps included in the smoothing interval. In this case, the value of $k$ is determined empirically. This approach is limited to the series level model as a random constant. The approach to the choice of the optimal smoothing parameter based on the Hurst indicator, calculated by TS, is set out in [17]. The main limitation of this approach is the complexity and availability of stationary TS segments for calculating the Hurst index, as well as the impossibility of applying the approach in real time. A self-learning model of short-term $\mathrm{F}$ of the socio-economic dynamics based on the apparatus of the theory of functions of complex variables is considered in [18]. An important advantage of this model in comparison with other modifications of ZOBM is that it does not require an a priori setting of the type of trend of the TS under study. However, the limitations of the model include its complexity and high sensitivity to the accuracy of the choice of the initial values for the complex smoothing coefficient. Improving the accuracy of the short-term $\mathrm{F}$ of a dangerous irreversible process in the form of FP based on the use of a modified ZOBM, in which an a priori model of the dynamics of the level of the studied TS for the corresponding fire hazards (FHs) is presented in [19].

Known PP F models are limited to deterministic processes to describe the basic FHs [20]. This means that the known FP F models are limited to the class of reversible deterministic PPs without taking into consideration the randomness factor. The scope of use of such models is limited mainly to the design stage and evaluation of the effectiveness of the proposed fire prevention measures. The real $\mathrm{F}$ of irreversible PPs and self-adjusting ZOBMs are not considered. However, study [21] notes that the real air environment in a fire is a complex dynamic system. Such a system is characterized by the properties of nonlinearity, self-organization, and dissipation. Ignoring these properties leads to significant errors in the assessment of the corresponding TS and PP F [22]. At the same time, [23] emphasizes that the current dynamics of FHs are of paramount importance for PP F. In this case, the technologies adopted in the nonlinear dynamics of complex systems are constructive [24]. Modern techniques of quantitative analysis of nonlinear dynamics of complex systems under non-stationary conditions are presented in [25]. However, in [24, 25], PP F on the basis of techniques of nonlinear analysis of the state of $\mathrm{FH}$ under actual conditions are not considered and not investigated. At the same time, there are several works in which experimental studies of the emergence and development of an irreversible process in the form of FP are considered. For example, in [26], the characteristics of the process of FP occurrence are experimentally investigated. 
Paper [27] reports the results of experimental studies of the effect of thermal radiation on the rate of heat generation by combustible materials. A detailed study of the rate of heat transfer at FP is performed in [28]. In [28], it is noted that the dynamics of the state of $\mathrm{FH}$ in the occurrence of FP is complex and non-stationary. Improving the efficiency of detection of FP based on known technologies is considered in [29]. At the same time, models of FP F based on the dynamics of the state of FHs are not investigated [30]. In [31], it is proposed to apply self-adjusting techniques for detecting fires under conditions of non-stationarity of FH states. The main limitation of those techniques is that self-adjustment is made on the basis of averaged values of individual parameters of the state of FHs. Taking into consideration the non-stationarity of the parameters, autocorrelations and pair correlations of FHs in the model chamber are investigated in [32]. At the same time, it is noted that the current values of FHs, and not their correlations, are more informative for detecting fires. Technologies suitable for identifying irreversible processes, based on the analysis of the current state of FHs, are considered in [33]. However, those techniques are limited to the consideration of a stationary model of $\mathrm{FH}$ states in the development of irreversible processes. Such a model limits the use of techniques [33] for the F of irreversible PPs under actual conditions. The technology of identifying irreversible processes under the conditions of non-stationary nature of FH states is considered in [34]. However, this technique is based on the use of the Fourier transform to stationary fragments of non-stationary dynamics of FH states. At the same time, it is difficult to isolate stationary fragments in the non-stationary dynamics of FH states in the event of an irreversible process. The technique of frequency-time identification of nonlinear systems for identifying the noted features of FHs is considered in [36, 37]. However, it is noted that those technologies are complex and do not allow them to be used for the F of irreversible PPs under actual conditions. In [38], the frequency-time technique for estimating dynamics of the state of FHs at ignition is considered in detail. It is noted that this technology is also quite complex and does not provide operational F of FPs. For operational F of FPs, it is proposed in [39] to use the ZOBM for the current measure of recurrence of increments of FH states. The advantage of this model is that on its basis, the F of TS with arbitrary level dynamics is possible. The quality of $\mathrm{F}$, in this case, depends on the value of the exponential smoothing parameter and the real dynamics of the level of the studied TS [40]. In [15], it is shown that to improve the quality of $\mathrm{F}$ of irreversible PPs, the classical set for the smoothing parameter is advisable to expand. Based on this, paper [41] reports a study into the quality of F of FP based on the use of ZOBM for the smoothing parameter from the classical and beyond-the-limit sets. It is confirmed that in the case of a beyond-the-limit set, the $\mathrm{F}$ quality indicators increase. However, it is noted that the quality of $\mathrm{F}$ is limited by the real dynamics of the irreversible process and the selected smoothing parameter in the ZOBM. At the same time, a self-adjusting ZOBM is not considered.

Thus, the F of irreversible PPs presents a particular difficulty, which is due to the lack or violation of the stability of the statistical characteristics for the TS of the PP indicators under consideration. The F of such PPs can be carried out on modifications of ZOBM. This model is adaptive and makes it possible, at certain values of the parameter $h$, to predict TS with arbitrary and a priori unknown dynamics of the level. When one selects the parameter $h$ from a beyond-the-limit set, one can predict TS with the dynamics of the level of evolutionary and chaotic nature. However, the accuracy of the forecast depends on the specific dynamics and the given value of the parameter $h$. The well-known self-learning model is designed for short-term forecasting of the particular class of PPs from the socio-economic area. At the same time, this model is complex and has a high sensitivity to the accuracy of setting the initial values for a complex smoothing coefficient. The self-adjusting model of the F of irreversible PPs with arbitrary and a priori unknown dynamics for forecasting FP is not considered and investigated in the cited sources. It is established that the dynamics of FH states in FP has a complex nonlinear character, depending on the real conditions of ignition. To detect fires, most of the known technologies are complex, have limited sensitivity and efficiency. For this reason, their use for the F of FP is problematic. It is noted that technologies based on techniques of nonlinear dynamics are constructive $[40,41]$. It is indicated that the preferred for the $\mathrm{F}$ of PPs are forecasting technologies based on ZOBM [40]. In this regard, an important and unresolved part of the problem under consideration is the construction of a self-adjusting ZOBM, which could be used to predict PPs under actual conditions.

\section{The aim and objectives of the study}

The aim of this work is to devise a self-adjusting zeroorder Brown's model for predicting irreversible processes and phenomena.

To accomplish the aim, the following tasks have been set:

- to theoretically substantiate the self-adjusting zero-order Brown's model, designed to predict irreversible processes and phenomena;

- to check the operability of the devised model using an example of the experimental time series of the given indicator, determined on the basis of the current values of the main fire hazards (smoke density, temperature, carbon monoxide concentration) for the air environment in the laboratory chamber when alcohol ignites.

\section{The study materials and methods}

The object of this study is a self-adjusting ZOBM for the F of irreversible PPs (including real FPs). The subject of our research is the experimental current errors in the $\mathrm{F}$ of PPs for the self-adjusting ZOBM when measuring the FHs of the air environment in the laboratory chamber during alcohol ignition. A detailed description of the materials, methods, and procedure of the original study is presented in [41]. We studied the current errors in the forecast of FPs one step ahead based on data from a laboratory experiment. The experiment involved the forced ignition of a test substance in the form of alcohol in a chamber imitating the corresponding non-airtight room. Combustible material was selected based on the fact that alcohol has a maximum ignition rate and makes it possible to simulate explosions in premises during a fire. Errors in $\mathrm{F}$ were estimated by the values of the current square and absolute F error. Data registration and processing were carried out using a PC. Specially developed software was used to record the data. Data processing was carried out on the basis of the software developed for the computational environment Mathcad 14 (USA). 


\section{The results of devising a self-adjusting model} for forecasting irreversible processes and phenomena

5. 1. Theoretical substantiation of the Brown's self-adjusting forecast model

ZOBM is widely used in practice for $\mathrm{F}$ various PPs. The widespread use of this model is due to its simplicity and clear physical meaning of the $\mathrm{F}$ procedure. Features of the use of ZOBM for the F of early fires and FPs are considered in [39]. An important advantage of ZOBM is the ability to adapt to destabilizing factors in the studied TS [42]. At the same time, the adaptability of the model and the accuracy of $\mathrm{F}$ the unknown level of the TS under consideration are determined by only one parameter - the model's smoothing parameter. The adaptability property of such a model is especially important in F both irreversible PPs as a whole and FPs. When igniting materials in the premises, the dynamics of the state of the air environment, determined by FH, are complex and of a priori not known random nature. Therefore, it is the ZOBM that should be considered more suitable for forecasting PPs. In general, by following a given model, the examined TS for an arbitrary air medium FH can be defined as:

$$
s_{i}=T_{i}+n_{i}
$$

where $T_{i}$ is an unknown and time-dependent on a discrete point $i$ level of TS for an arbitrary $\mathrm{FH} ; n_{i}$ is the value of random noise at moment $i$.

For TS (1), ZOBM can be represented as:

$$
P_{i}(d)=\widehat{T}_{i},
$$

where $F_{i}(d)$ is the $\mathrm{F}$ of TS of the unknown level (1) at the moment $i+d$ executed at moment $i$; $\widehat{T}_{i}$ is the estimation at the moment $i$ of the time of the level of TS (1) for an arbitrary FH.

In ZOBM (2), an exponentially smoothed mean value for current TS measurements (1) is usually used as an estimate of $\widehat{T}_{i}$, determined from the following recurrent formula:

$$
\widehat{T}_{i}=h s_{i}+(1-h) \widehat{T}_{i-1}
$$

where $h$ is the exponential smoothing parameter; $s_{i}$ is the measured value of TS (1) at moment $i ; \widehat{T}_{i-1}$ is the exponentially smoothed mean TS (1) at a discrete time $i-1$ (F at time $i-1$ ).

For the $\mathrm{F}$ of irreversible PPs, the short-term forecast is of particular interest - that is, F one step ahead. That means that for the F of irreversible PPs, in expression (2), the value of $d=1$. It should be noted that in this case, the F of FP will be based only on exponentially smoothed average values of TS (1), the error of which, in turn, will depend on a single parameter $h$ of smoothing. In this case, the error in $\mathrm{F}$ the FP will be completely determined by the error of exponential smoothing, as well as the error of inconsistency of model (1) with the real TS, determined by the corresponding indicators of FH. In accordance with expression (3), F for the current observation step is determined using the previous forecast and the current value of the series, which are pre-weighed with the weights determined in accordance with the specified parameter $h$ of smoothing. $h$ is often interpreted as a parameter that determines the adaptive properties of ZOBM. At $h=0$, the model is non-adaptive. In this case, the forecast value of the TS for the current measurement step coincides with the forecast value of the series in the previous step. At $h=1-\mathrm{ZOBM}$ is fully adaptive. In this case, the predicted value of the series for the current measurement step coincides with the measured value of the series at the current step. In [43], it is noted that the greater the value of $h$, the greater the contribution of the latter measurements to the formation of the exponential mean. The influence of the initial conditions of $\mathrm{F}$ on the formation of the exponential mean, in this case, decreases rapidly. With a small value of the parameter $h$, the exponential mean is formed taking into consideration a large number of past measurements. In this case, the influence of past measurements on the exponential mean decreases much more slowly.

That means that the considered ZOBM (2), taking into consideration (3), when choosing the smoothing parameter from the classical set, is designed for the $\mathrm{F}$ of predominantly homogeneous stationary TS and, therefore, is not suitable for the F of irreversible PPs. Various modifications of ZOBM are known, expanding its capabilities for the case of $\mathrm{F}$ the non-stationary series. For example, it is stated in $[10,11]$ that for the F of non-stationary series, it is necessary to modify ZOBM (2) taking into consideration recurrent formula (3) by selecting the parameter $h$ from the beyond-the-limit set from 1 to 2 . It should be noted that the recurrent formula of exponential smoothing (3) can be written in another equivalent form:

$$
\widehat{T}_{i}=\widehat{T}_{i-1}+h\left(s_{i}-\widehat{T}_{i-1}\right) .
$$

Then expression (4) will resemble the known recurrent formula for the optimal discrete Kalman filter for the case of observations $s_{i}$. In this case, the difference of (4) from the formula of the optimal discrete Kalman filter is that the variable gain factor in (4) is replaced by a fixed value corresponding to the specified value of $h$. Therefore, such a filter is non-stationary, and representation (4) describes a stationary filter. If one selects $h$ equal to the steady value of the non-stationary gain of the optimal discrete Kalman filter, such a filter becomes quasi-optimal. It is known that the Kalman filter provides the best accuracy of the assessment. At the same time, in the class of linear devices, such a filter provides the absolute best estimate. However, this property is provided only if the specified conditions are fully complied with the real ones.

Applying Kalman's approach to (4), in this case, means that for TS (1) in the ZOBM, the level of the $T_{i}$ series is determined by a model of the following form:

$$
T_{i}=T_{i-1}
$$

That means that (5) sets the current level of TS (1) as a random constant. The initial value of the level of the series $T_{0}$ in this case obeys the Gaussian distribution with the mathematical expectation $T_{0}$ and the known variance $\sigma T_{0}^{2}$. If the actual level of the series (1) differs from model (5), the Kalman filter (4) will not provide its optimal assessment. In this case, following Kalman's approach, the $h$ parameter in (4) should depend on the step number. In this case, the dispersion of random noise $n_{i}$ in (1) must be known. Let the random noise $n_{i}$ in (1) follow a Gaussian distribution with zero mean and fixed variance $\sigma^{2}$. Given these conditions, the optimal $h o_{i}$ parameter in (4) at each step will be determined as:

$$
h o_{i}=\frac{\sigma T_{i}^{2}}{\sigma^{2}}
$$

where $\sigma T_{i}^{2}$ is the variance of estimating the level of the series at time $i$. 
It can be assumed that in this case, $\sigma T_{i}^{2}$ will be less than the value of $\sigma^{2} / i$. This means that with an increase in the observation time or the number of measurements, the accuracy of estimating the level of TS tends to zero. However, this result is only for a particular model of the level of a series of form (5). For the dynamics of the level (arbitrary indicator) of the TS of a general nature, this does not hold. In the theory of discrete linear filtration by Kalman, a general model described by the difference equation is considered:

$$
T_{i}=\Phi_{i, i-1} T_{i-1}+\Gamma_{i-1} q_{i-1},
$$

where $\Phi_{i, i-1}$ is the known transient state function; $\Gamma_{i-1}$ is a known non-random sequence; $q_{i-1}$ is a random uncorrelated Gaussian sequence with zero mean and unit variance.

Following Kalman's approach, in this case, the difference equation of observation will be described by TS (1) of the discrete states of the medium determined by an arbitrary FH [44]. This approach assumes that the random discrete sequence $n_{i}$ of noise in (1) is independent of a random discrete sequence in (7) [45] and is an uncorrelated Gaussian sequence with zero mean and the specified dispersion $\sigma_{i}^{2}$.

Important for $\mathrm{F}$ is the recurrent formula that determines the gain of the Kalman filter. This is explained by the fact that the recurrent gain of the discrete Kalman filter provides optimal estimation (7) and accuracy of $\mathrm{F}$ for the ZOBM under consideration. For the case of the specified equations (7) and (1), the optimal gain $\mathrm{K}_{i}$ of the discrete Kalman filter is non-stationary:

$$
\mathrm{K}_{i}=\frac{\eta_{i, i-1}}{\eta_{i, i-1}+\sigma_{i}^{2}} .
$$

where $\eta_{i, i-1}=\mathrm{M}\left(\left(T_{i}-\widehat{T}_{i}\right)\left(T_{i-1}-\widehat{T}_{i-1}\right)\right)$ is the covariance of the estimation error for the corresponding discrete steps; $\mathrm{M}\left({ }^{*}\right)$ is a mathematical expectation operator. It follows from (8) that the current value of $\mathrm{K}_{i}$ depends on the corresponding covariance of the current estimation error and the current variance in the measurement noise. Thus, for the case of low noise variance of measurement, the value of the optimal gain is close to 1 . This means that the single gain extrapolation estimate $\Phi_{i, i-1} \widehat{T}_{i-1}$ is corrected by a signal $\left(s_{i}-\Phi_{i, i-1} \widehat{T}_{i-1}\right)$, representing an error between the extrapolated estimate and the new measurement. At the current variance in measurement noise significantly exceeding the current covariance of the estimation error, the value of the optimal gain tends to zero. In this case, the above considered correction of the extrapolated estimate is practically not performed, and the assessment is carried out in accordance with the specified equation for the dynamics of the level (7). In the case of ZOBM, equation (7) is unknown. At the same time, the current variance of the measurement noise in (1) is also unknown. Therefore, it is not possible to use directly the results of optimal discrete Kalman filtration. Under these conditions, it is possible to increase the accuracy of $\mathrm{F}$ based on the corresponding modification of the ZOBM by additionally introducing an equation for the dynamics of the level of the studied series. For example, in [19], it is proposed to modify ZOBM, supplementing it with a difference equation that takes into consideration both the random level of the series and the increment of the series level at adjacent discrete moments. It is shown that such a modification of ZOBM allows for an order of magnitude increase in the accuracy of short-term forecasting of dynamic processes and phenomena with a sharp change in dynamics, characteristic, for example, of explosions, rapid fires, and sudden failures of controlled equipment. In this case, the proposed modification is limited to an a priori model of the predicted process. If the actual predicted process differs from the a priori model, then the $\mathrm{F}$ error may be unacceptably large. The $h$ ZOBM parameter, in this case, remains fixed for different time points. Therefore, it is required to be set in advance and thereby determine the adaptive capabilities of the proposed modification. The constancy of the smoothing parameter over time limits the adaptive properties of the modified ZOBM in case of difference between the real dynamics of the process and that specified a priori.

The application of Kalman's approach to the modification of ZOBM, which differs from [19], is that the model of the predicted process is not set a priori, and the $h$ parameter is not fixed but is chosen as a variable for various discrete time moments. Since the a priori process model is not specified, it is not possible to determine the optimal gain $\mathrm{K}_{i}$ of the discrete Kalman filter according to expression (8). In addition, it is also not possible to determine $\mathrm{K}_{i}$ since the variance of measurement noise in (1) is also unknown and can change over time in an arbitrary way. However, the measured value is $z_{i}=s_{i}-\widehat{T}_{i-1}$, which contains current information about the resulting $\mathrm{F}$ error, due to the difference in the real dynamics of the level of the series from its assessment and the actual noise of the measurement. At the same time, a feature of the value $z_{i}=s_{i}-\widehat{T}_{i-1}$ is that it is not possible to identify the sources of the appearance of these errors in the resulting error. Therefore, the value $z_{i}=s_{i}-\widehat{T}_{i-1}$ can be used to determine the current value of the optimal gain of the discrete Kalman filter in the denominator of (8). However, in the case where the current measurement is close to the current estimate (forecast) of the level of the TS being studied, and the current noise is small, the gain of the discrete Kalman filter would increase significantly. That would lead to the divergence of the Kalman filter and to an increase in the estimation error and, accordingly, in F [45]. In order to eliminate this divergence, it is proposed to determine the current gain $\mathrm{K}_{i}$ in accordance with the following expression:

$$
\mathrm{PK}_{i}=\frac{1}{1+z_{i}^{2}} \text {. }
$$

Taking into consideration (9), the proposed discrete Kalman filter will no longer be optimal but will be suboptimal:

$$
\widehat{T}_{i}=\widehat{T}_{i-1}+\mathrm{PK}_{i}\left(s_{i}-\widehat{T}_{i-1}\right) .
$$

Expression (10) will describe the proposed recurrent formula for self-adjusting exponential smoothing in a self-adjusting ZOBM. This formula, together with expression (2), will generally define the proposed ZOBM for the F of irreversible PPs, obtained through the application of the Kalman approach. The proposed ZOBM does not require setting an adaptation parameter. In addition, a given model allows the F of not only FPs but also time series generated by unknown types of irreversible PPs, taking into consideration the effect of arbitrary non-stationary noises.

5. 2. Verifying the feasibility of the proposed Brown's self-adjusting predictive model

The operability of the proposed self-adjusting ZOBM for the F of PPs was checked on the basis of the experimental TS of the recurrence of increments of the vector of air medium 
states in the chamber during alcohol ignition. As a vector of air medium states, a vector was considered, the elements of which were determined by three main FHs in the form of smoke density, temperature, and carbon monoxide concentration, measured by appropriate sensors [41].

Fig. 1 shows the dynamics in the $\mathrm{F}$ of the recurrence of increments of the vector of states of the air medium obtained on the basis of a self-adjusting ZOBM (auto). For comparison, it demonstrates the dynamics in the $\mathrm{F}$ of the recurrence of the increment vector of the air medium states for the case of using the known ZOBM with a fixed smoothing parameter from the classical $(h=0.2)$ and beyond-the-limit $(h=1.2)$ sets.

Black color indicates the studied TS of the recurrence of increments of the vector of states of the air medium. Fig. 2 shows the dynamics of the forecast error determined by the current square of SE (Squared Error) error for the selfadjusting ZOBM and ZOBM with a fixed smoothing parameter from the classical and beyond-the-limit sets.

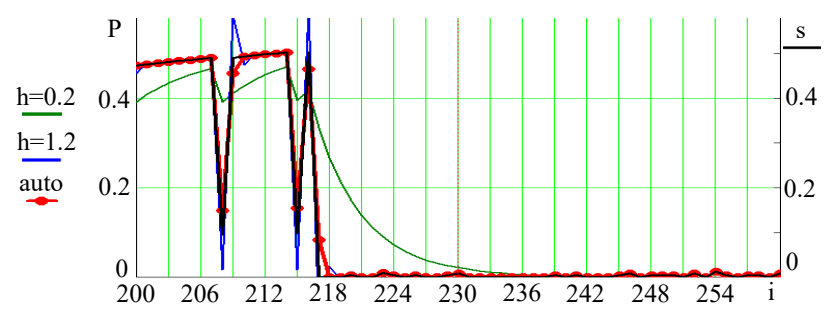

Fig. 1. Dynamics of forecast of recurrence of increments of vector of states of air medium in a laboratory chamber

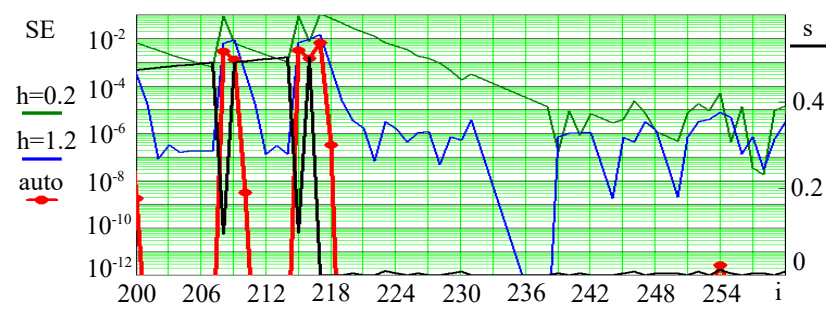

Fig. 2. Dynamics of prediction errors determined by the current error squares for the studied Brown's models of zero order

An illustration of the corresponding dynamics of the forecast error, determined by the absolute error AE (Absolute Error), for the considered self-adjusting ZOBM and ZOBM with a fixed smoothing parameter from the classical and beyond-the-limit sets is shown in Fig. 3.

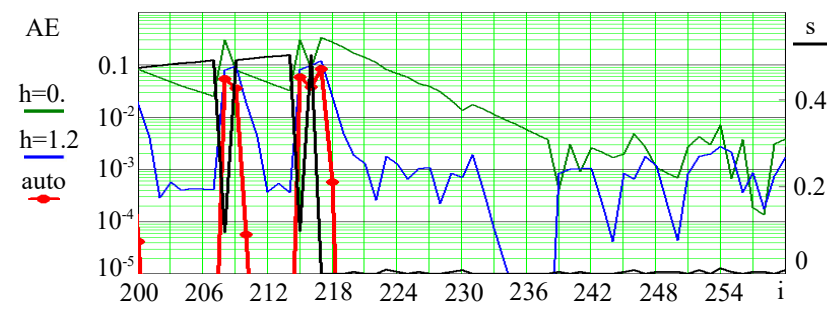

Fig. 3. Dynamics of prediction errors determined by the current absolute errors for the Brown's zero-order models under study

Designations and initial data given in Fig. 2, 3 are identical to the designations and initial data used in Fig. 1.

\section{Discussion of results of the feasibility of the proposed self-adjusting forecast Brown's model}

The results (Fig. 1) of the experimental study of the dynamics of $\mathrm{F}$ in the test irreversible process (ignition of alcohol) are explained by the advantage of the self-adjusting ZOBM in comparison with the considered known modifications given in Fig. 1. This is due to the fact that in the proposed model the exponential smoothing parameter is non-stationary. For each discrete moment, the value of the parameter is determined by the current resulting error, depending on the real dynamics of the series level and the current noise. Our analysis of curves in Fig. 1 reveals that in this case, the best $\mathrm{F}$ is provided by the proposed ZOBM. The worst $\mathrm{F}$, in this case, is produced by ZOBM for the case of a fixed smoothing parameter from the classical set. The intermediate quality of $\mathrm{F}$ is demonstrated by ZOBM for the case of a fixed smoothing parameter from the beyond-the-limit set. At the same time, one can see that forecast errors are maximum in the event of a sharp change in the dynamics of the TS level. A further increase in the smoothing parameter leads to an increase in the $\mathrm{F}$ error of the jump-like dynamics of the series level. Fig. 2 illustrates quantitative estimates of the quality of the forecast in the form of the current dynamics of the error, determined by the square of its values for the types of ZOBM under consideration. For the proposed ZOBM, the current square of the F error for the experimental TS under study is more than six orders of magnitude smaller compared to the case of choosing a fixed smoothing parameter from a beyond-the-limit set. And for the case of abrupt changes in the dynamics of the level of the series, on average, it turns out to be half as much. It should be noted that it is the abrupt change in the dynamics of the studied time series of the recurrence of the increments of the state of $\mathrm{FH}$ that is a sign of the beginning of the ignition of alcohol and, therefore, is important for PPs. The use of ZOBM with the smoothing parameter from the classical and beyond-the-limit sets for FPs would lead to the omission of the beginning of the ignition of alcohol, which could lead to the development of a fire [41].

The quantitative estimates of the quality of $\mathrm{F}$ in the form of the current dynamics of the error, determined by its absolute values for the types of ZOBM under consideration, are shown in Fig. 3. At the same time, the nature of the current values of the absolute F error for the models under consideration is generally similar to Fig. 2 .

Thus, the experimental data testify, in general, to the feasibility of the proposed self-adjusting ZOBM. At the same time, this model provides high accuracy of FP forecast and also allows the $\mathrm{F}$ of irreversible PPs with unknown dynamics. In addition, the self-adjusting ZOBM does not require a complex way to select the smoothing parameter for specific TS dynamics. This means that the proposed ZOBM provides an accurate and accurate forecast of processes with unknown a priori dynamics, including random and chaotic processes.

The limitations of the original study include the fact that the results of the test of the self-adjusting ZOBM are given for the experimental data on the state of the air environment in a laboratory chamber during alcohol ignition. It should also be noted that the results obtained were influenced by the parameters of the chamber, the size of the ignition site, and the placement of the measuring sensors of FHs. Further development of this research could involve similar experimental studies in relation to actual PPs. Such studies would need to assess the practical limits of applicability, errors in F, and possible limitations of the proposed self-adjusting ZOBM. 


\section{Conclusions}

1. A zero-order self-adjusting Brown's model has been theoretically substantiated. The model makes it possible to predict with high accuracy not only fires but also irreversible processes and phenomena of a random and chaotic nature under actual conditions. Forecasting such processes and phenomena is proposed to be carried out for the time series of the indicator in the form of a measure of the recurrence of increments of the state of hazardous factors. The essence of the devised model is that, based on Kalman's approach, the smoothing parameter is proposed to be set for each time moment. Such a parameter is determined depending on the resulting current forecast error, taking into consideration the real and unknown dynamics of the studied series and noise. That has made it possible to abandon the complex selection of the smoothing parameter for the specific dynamics of the study series. The proposed Brown's model, unlike the known modifications, does not require setting a dynamics model of the level of the time series under study, provides negligible errors, and efficiency of the forecast.
2. The operability of the zero-order self-adjusting Brown's model was tested on the example of the experimental time series for the current measure of the recurrence of the increments of the state of the air medium in a laboratory chamber during alcohol ignition. As quantitative indicators of the quality of the forecast error, their current values for the square and absolute values are considered. It has been established that for the proposed model, the current square of the prediction error for the experimental time series under study is more than six orders of magnitude smaller compared to the case of choosing a fixed smoothing parameter from a beyond-the-limit set. At the same time, the current square of the forecast error for abrupt changes in the dynamics of the series level on average is half as small as the fixed parameter of the beyond-the-limit set. The results obtained indicate, in general, the efficiency of the proposed model. At the same time, the model makes it possible to predict irreversible processes and phenomena with unknown dynamics. In addition, a given model does not require the selection of the smoothing parameter, which provides an operational and accurate forecast of processes with unknown a priori dynamics, including random and chaotic processes.

\section{References}

1. Migalenko, K., Nuianzin, V., Zemlianskyi, A., Dominik, A., Pozdieiev, S. (2018). Development of the technique for restricting the propagation of fire in natural peat ecosystems. Eastern-European Journal of Enterprise Technologies, 1 (10 (91)), 31-37. doi: https://doi.org/10.15587/1729-4061.2018.121727

2. Vambol, S., Vambol, V., Kondratenko, O., Koloskov, V., Suchikova, Y. (2018). Substantiation of expedience of application of high-temperature utilization of used tires for liquefied methane production. Journal of Achievements in Materials and Manufacturing Engineering, 2 (87), 77-84. doi: https://doi.org/10.5604/01.3001.0012.2830

3. Vambol, S., Vambol, V., Sobyna, V., Koloskov, V., Poberezhna, L. (2019). Investigation of the energy efficiency of waste utilization technology, with considering the use of low-temperature separation of the resulting gas mixtures. Energetika, 64 (4), 186-195. doi: https://doi.org/10.6001/energetika.v64i4.3893

4. Semko, A., Rusanova, O., Kazak, O., Beskrovnaya, M., Vinogradov, S., Gricina, I. (2015). The use of pulsed high-speed liquid jet for putting out gas blow-out. The International Journal of Multiphysics, 9 (1), 9-20. doi: https://doi.org/10.1260/1750-9548.9.1.9

5. Vambol, S., Vambol, V., Kondratenko, O., Suchikova, Y., Hurenko, O. (2017). Assessment of improvement of ecological safety of power plants by arranging the system of pollutant neutralization. Eastern-European Journal of Enterprise Technologies, 3 (10 (87)), 63-73. doi: https://doi.org/10.15587/1729-4061.2017.102314

6. Otrosh, Y., Semkiv, O., Rybka, E., Kovalov, A. (2019). About need of calculations for the steel framework building in temperature influences conditions. IOP Conference Series: Materials Science and Engineering, 708, 012065. doi: https://doi.org/10.1088/ 1757-899x/708/1/012065

7. Dadashov, I., Loboichenko, V., Kireev, A. (2018). Analysis of the ecological characteristics of environment friendly fire fighting chemicals used in extinguishing oil products. Pollution Research, 37 (1), 63-77.

8. Lukashin, Yu. P. (2003). Adaptivnye metody kratkosrochnogo prognozirovaniya vremennyh ryadov. Moscow: Finansy i statistika, 416.

9. Brown, R. G. (2004). Smoothing, forecasting and prediction of discrete time series. Dover Publications, 480.

10. Svetun'kov, S. G., Butuhanov, A. V., Svetun'kov, I. S. (2006). Zapredel'nye sluchai metoda Brauna v ekonomicheskom prognozirovanii. Sankt-Peterburg: SPbGUEF, 71.

11. Hyndman, R. J., Khandakar, Y. (2008). Automatic time series forecasting: the forecast Package for R. Journal of statistical software, 27 (3), 1-22. doi: https://doi.org/10.18637/jss.v027.i03

12. Gambarov, G. M., Zhuravel', N. M., Korolev, Yu. G. (1990). Statisticheskoe modelirovanie i prognozirovanie. Moscow: Finansy i statistika, 383.

13. Chetyrkin, E. M. (1977). Statisticheskie metody prognozirovaniya. Moscow: Statistika, 200.

14. Lugachev, M. I., Lyapuntsov, Yu. P. (1999). Metody sotsial'no-ekonomicheskogo prognozirovaniya. Moscow: TEIS, 160.

15. Svetun'kov, S. G. (2002). O rasshirenii granits primeneniya metoda Brauna. Izvestiya Sankt-Peterburgskogo gosudarstvennogo universiteta ekonomiki i finansov, 3, 94-107.

16. Vartanyan, V. M., Romanenkov, Yu. A., Kononenko, A. V. (2005). Parametricheskiy sintez prognoznoy modeli eksponentsial'nogo sglazhivaniya. Vestnik NTU «KhPI», 59, 9-16.

17. Tebueva, F., Streblianskaia, N. (2016). Adaptive method for predicting short time series of natural processes. Sovremennaya nauka: aktual'nye problemy teorii i praktiki, 6, 83-87.

18. Svetun'kov, I. S. Samoobuchayuschayasya model' kratkosrochnogo prognozirovaniya sotsial'no-ekonomicheskoy dinamiki. Available at: https://www.hse.ru/data/2011/02/28/1211522815/2010_mk_article.pdf 
19. Pospelov, B., Rybka, E., Krainiukov, O., Yashchenko, O., Bezuhla, Y., Bielai, S. et. al. (2021). Short-term forecast of fire in the premises based on modification of the Brown's zero-order model. Eastern-European Journal of Enterprise Technologies, 4 (10 (112)), 52-58. doi: https://doi.org/10.15587/1729-4061.2021.238555

20. Koshmarov, Yu. A., Puzach, S. V., Andreev, V. V. (2012). Prognozirovanie opasnyh faktorov pozhara v pomeschenii. Moscow: AGPS MChS Rossii, 126.

21. Pospelov, B., Andronov, V., Rybka, E., Meleshchenko, R., Borodych, P. (2018). Studying the recurrent diagrams of carbon monoxide concentration at early ignitions in premises. Eastern-European Journal of Enterprise Technologies, 3 (9 (93)), $34-40$. doi: https://doi.org/10.15587/1729-4061.2018.133127

22. Andronov, V., Pospelov, B., Rybka, E., Skliarov, S. (2017). Examining the learning fire detectors under real conditions of application. Eastern-European Journal of Enterprise Technologies, 3 (9 (87)), 53-59. doi: https://doi.org/10.15587/1729-4061.2017.101985

23. Ahn, C.-S., Kim, J.-Y. (2011). A study for a fire spread mechanism of residential buildings with numerical modeling. WIT Transactions on the Built Environment, 117, 185-196. doi: https://doi.org/10.2495/safe110171

24. Webber, C. L., Ioana, C., Marwan, N. (Eds.) (2016). Recurrence Plots and Their Quantifications: Expanding Horizons. Springer Proceedings in Physics. doi: https://doi.org/10.1007/978-3-319-29922-8

25. Sadkovyi, V., Pospelov, B., Andronov, V., Rybka, E., Krainiukov, O., Rud, A. et. al. (2020). Construction of a method for detecting arbitrary hazard pollutants in the atmospheric air based on the structural function of the current pollutant concentrations. Eastern-European Journal of Enterprise Technologies, 6 (10 (108)), 14-22. doi: https://doi.org/10.15587/1729-4061.2020.218714

26. Poulsen, A., Jomaas, G. (2011). Experimental Study on the Burning Behavior of Pool Fires in Rooms with Different Wall Linings. Fire Technology, 48 (2), 419-439. doi: https://doi.org/10.1007/s10694-011-0230-0

27. Zhang, D., Xue, W. (2010). Effect of heat radiation on combustion heat release rate of larch. Journal of West China Forestry Science, 39, 148

28. Peng, X., Liu, S., Lu, G. (2005). Experimental Analysis on Heat Release Rate of Materials. Journal of Chongqing University, 28 , 122.

29. Andronov, V., Pospelov, B., Rybka, E. (2017). Development of a method to improve the performance speed of maximal fire detectors. Eastern-European Journal of Enterprise Technologies, 2 (9 (86)), 32-37. doi: https://doi.org/10.15587/1729-4061.2017.96694

30. Pospelov, B., Andronov, V., Rybka, E., Meleshchenko, R., Gornostal, S. (2018). Analysis of correlation dimensionality of the state of a gas medium at early ignition of materials. Eastern-European Journal of Enterprise Technologies, 5 (10 (95)), 25-30. doi: https://doi.org/10.15587/1729-4061.2018.142995

31. Pospelov, B., Andronov, V., Rybka, E., Skliarov, S. (2017). Research into dynamics of setting the threshold and a probability of ignition detection by selfadjusting fire detectors. Eastern-European Journal of Enterprise Technologies, 5 (9 (89)), $43-48$. doi: https://doi.org/10.15587/1729-4061.2017.110092

32. Pospelov, B., Rybka, E., Meleshchenko, R., Gornostal, S., Shcherbak, S. (2017). Results of experimental research into correlations between hazardous factors of ignition of materials in premises. Eastern-European Journal of Enterprise Technologies, 6 (10 (90)), 50-56. doi: https://doi.org/10.15587/1729-4061.2017.117789

33. Bendat, J. S., Piersol, A. G. (2010). Random data: analysis and measurement procedures. John Wiley \& Sons. doi: https://doi.org/ $10.1002 / 9781118032428$

34. Singh, P. (2016). Time-frequency analysis via the fourier representation. HAL, 1-8. Available at: https://hal.archives-ouvertes.fr/ hal-01303330/document

35. Pretrel, H., Querre, P., Forestier, M. (2005). Experimental Study Of Burning Rate Behaviour In Confined And Ventilated Fire Compartments. Fire Safety Science, 8, 1217-1228. doi: https://doi.org/10.3801/iafss.fss.8-1217

36. Stankovic, L., Dakovic, M., Thayaparan, T. (2014). Time-frequency signal analysis. Kindle edition, 655.

37. Giv, H. H. (2013). Directional short-time Fourier transform. Journal of Mathematical Analysis and Applications, 399 (1), $100-107$. doi: https://doi.org/10.1016/j.jmaa.2012.09.053

38. Pospelov, B., Andronov, V., Rybka, E., Popov, V., Semkiv, O. (2018). Development of the method of frequencytemporal representation of fluctuations of gaseous medium parameters at fire. Eastern-European Journal of Enterprise Technologies, 2 (10 (92)), $44-49$. doi: https://doi.org/10.15587/1729-4061.2018.125926

39. Pospelov, B., Andronov, V., Rybka, E., Samoilov, M., Krainiukov, O., Biryukov, I. et. al. (2021). Development of the method of operational forecasting of fire in the premises of objects under real conditions. Eastern-European Journal of Enterprise Technologies, 2 (10 (110)), 43-50. doi: https://doi.org/10.15587/1729-4061.2021.226692

40. Sinaga, H., Irawati, N. (2020). A Medical Disposable Supply Demand Forecasting By Moving Average And Exponential Smoothing Method. Proceedings of the Proceedings of the 2nd Workshop on Multidisciplinary and Applications (WMA) 2018, 24-25 January 2018, Padang, Indonesia. doi: https://doi.org/10.4108/eai.24-1-2018.2292378

41. Pospelov, B., Rybka, E., Meleshchenko, R., Krainiukov, O., Biryukov, I., Butenko, T. et. al. (2021). Short-term fire forecast based on air state gain recurrence and zero-order brown model. Eastern-European Journal of Enterprise Technologies, 3 (10 (111)), $27-33$. doi: https://doi.org/10.15587/1729-4061.2021.233606

42. Pospelov, B., Rybka, E., Togobytska, V., Meleshchenko, R., Danchenko, Y., Butenko, T. et. al. (2019). Construction of the method for semi-adaptive threshold scaling transformation when computing recurrent plots. Eastern-European Journal of Enterprise Technologies, 4 (10 (100)), 22-29. doi: https://doi.org/10.15587/1729-4061.2019.176579

43. Pospelov, B., Andronov, V., Rybka, E., Krainiukov, O., Karpets, K., Pirohov, O. et. al. (2019). Development of the correlation method for operative detection of recurrent states. Eastern-European Journal of Enterprise Technologies, 6 (4 (102)), 39-46. doi: https://doi.org/10.15587/1729-4061.2019.187252

44. Bestuzhev-Lada, I. V. (1982). Rabochaya kniga po prognozirovaniyu. Moscow: Mysl', 430.

45. Seydzh, E. P., Uayt, Ch. S. (1982). Optimal'noe upravlenie sistemami. Moscow: Radio i svyaz', 392. 